\title{
A Side-by-Side Study of 20 Consecutive FUE Patients Comparing the Use of a $0.9 \mathrm{~mm}$ Sharp vs. $0.9 \mathrm{~mm}$ Blunt Punch
}

David Josephitis, DO, FISHRS, Ron Shapiro, MD, FISHRS Minneapolis, Minnesota, USA drjosephitis@shapiromedical.com

\section{Introduction}

Follicular unit extraction (FUE) has come a long way over the past 15 years. While there is still controversy surrounding when it is best to use FUE, FUT, or a combination of both, there is no question that FUE is an excellent alternative for many situations. Much of this gained acceptance is due to improvements and modifications of the FUE technique that have been associated with improved graft quality and yield. There are now many variations of techniques that exist. Still, though, there is much needed research comparing the clinical significance of some of these different techniques.

There are many different types of FUE devices spanning from manual to motorized to robotic. Within these devices, there are various types of punch tip movements from rotational to oscillatory. It is challenging to find a practical way to study and compare all of these variables. One factor that is common to all of these devices, though, is that the punch used can be either sharp or blunt. The relative benefits of a sharp versus blunt punch have been the subject of much controversy. In this study, sharp and blunt punches are compared with respect to the following parameters: 1) hair transection rate, 2) hairs per graft, 3) yield/ missing grafts, 4) speed, 5) graft quality, and 6) scarring.

\section{Study Design}

Twenty consecutive patients undergoing FUE were enrolled in the study. They were all male and had never before had a hair transplant procedure. The recipient area planning was done as usual and the donor area was prepped by shaving. A vertical line was drawn in the midline of the patient's donor region extending from the superior border of the "safe" donor area to the most inferior border. A horizontal line was drawn along the superior region of the safe zone, perpendicular to the top of the first line. Two more lines were drawn parallel to the first midline demarcation $3 \mathrm{~cm}$ in width. This created two equal boxes $3 \mathrm{~cm}$ in width on both sides of the midline (Figure 1). A tattoo was placed in the center of each box and pre-study standard and macro photographs were taken of the donor area.

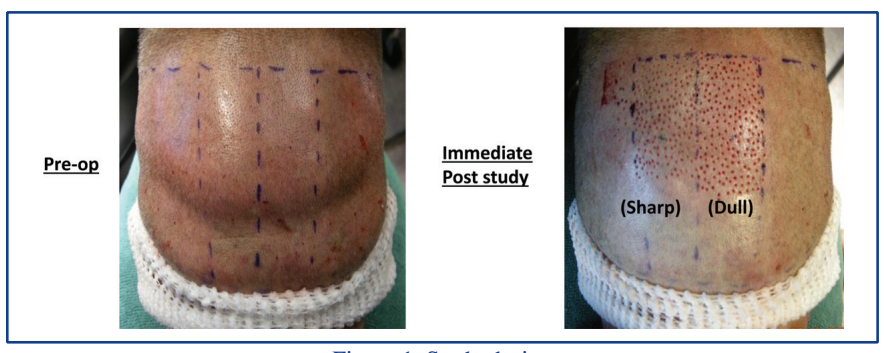

Figure 1. Study design

In all cases, the same physician experienced in both techniques performed 200 FUE extractions in each box at an extraction density of approximately $18 \mathrm{FU} / \mathrm{cm}^{2}$. A $0.9 \mathrm{~mm}$ Cole Instruments Serrounded Punch was used on the patient's left side and a $0.9 \mathrm{~mm}$ Harris SAFE System blunt punch was used on the patient's right side. The total time for extractions was recorded for both boxes. The time for scoring the graft (punching) and extracting the graft (pulling) were recorded separately. During extraction, the number of capped grafts was also documented. A "dedicated" technician recorded the number of each type of graft (1's, 2's, 3 's, etc.) along with noting any transections. ALL transections, no matter how small, were recorded, and no trimming of grafts was done at this point. Macro photographs were taken of the grafts after they were extracted. Only after all the data was collected were the grafts passed on to the other technicians to be further processed in the normal manner. All calculations were performed on the collected data at the end of the day of surgery.

There were 5 patients who were able to return for follow-up photos of their donor area between 5 and 10 months after surgery. Standard and macro photography of their shaved donor area at the sites of the tattoos were repeated in order to assess differences in scarring between the two types of punches.

\section{Results}

Hair Transection Rate (Transected Hairs/Total Hairs)

The data indicated that there was a greater percentage of transection with the sharp punch compared to the blunt punch (23.6\% sharp vs. $9.7 \%$ blunt) (Figure 2). In order to better understand the significance of the transection that was occurring, in 5 patients an extra step was taken to record the percentage of transection that occurred at various levels along the hair shaft. Six different types (levels) of transection were seen (Figure 3). Of the transections, $22 \%-36 \%$ were Type 6 , which was only a tiny spicule at the upper portion of the dermis. Although it was recorded, most physicians would likely agree that this type of transection is probably not clinically significant as greater than two-thirds of the hair was left in vivo and would most likely regrow. However, about $60 \%$ of the transections were a Type 4 or 5 with the transection being somewhere near the bulge or center of the shaft. The fates of these transections are more controversial and may indeed be significant. ${ }^{1,2}$

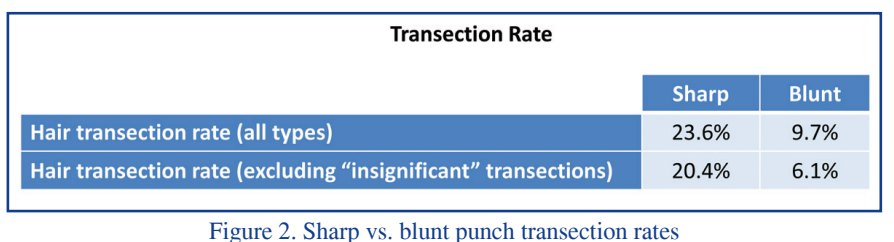

If the transection rate is adjusted to exclude the Type 6 (probably insignificant) transections, the hair transection rate decreases slightly for both the sharp and blunt punches. (20.4\% sharp vs. $6.1 \%$ blunt). The sharp punch remained having a greater number of transections (Figure 2).

\section{Hairs per Graft}

The findings show that both the sharp and the blunt punch produced almost identical hairs/graft rates ( 2.5 hairs/graft sharp vs. 2.6 hairs/graft blunt) (Figure 4). Initially, there was doubt cast on the accuracy and reliability of these rates as the transection rates had already been found to be different. Under closer scrutiny, the numbers revealed the answer. When originally calculating hairs/graft, equal weight was given to ALL of the 
hairs in each graft. There was no distinguishing between the transected and non-transected hairs within each graft. For example, a graft with 3 hairs in it would be recorded as a 3-hair graft of the hairs in that graft were transected. This is an important point, because it has been increasingly suggested by some FUE physicians that the only parameter

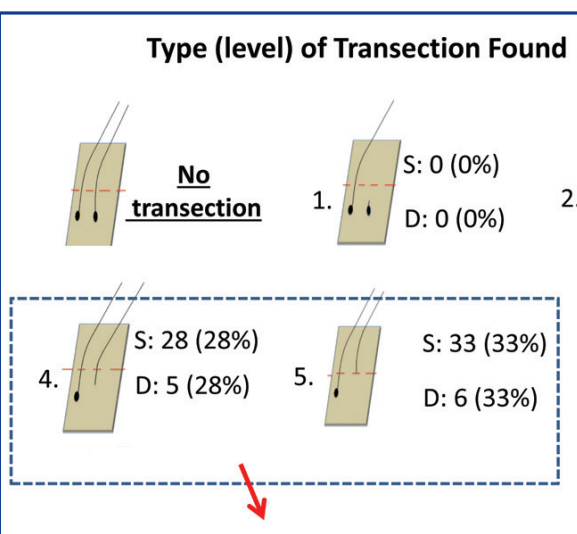

Over $60 \%$ of Transection was Type 4 or Type 5

\section{In 5 Patients (2000 Grafts)}
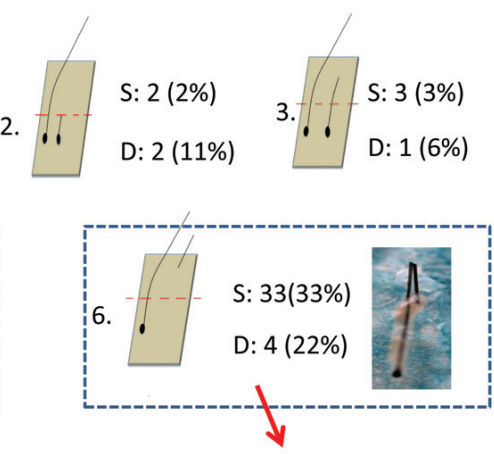

Level 6 Probably Insignificant?
Figure 3. Levels of transection no matter if 1,2 , or all 3

was identical between the two punches at $5 \%$. Initially, it was assumed that the sharp punch would have had a higher rate of capping because intrinsically the depth of a sharp punch is typically more limited than a blunt punch. After eliminating the number of capped grafts from the equation, it was shown that the blunt punch still had a higher number of physicians need to look at to evaluate the overall success of their FUE extractions is the hairs/graft parameter, and that the hair transection rate is not as important. With the current body of knowledge, this is a premature and dangerous assumption. While it is true that transected hair has the potential to grow back, many studies have shown varying degrees of decreased survival with transected hairs. The survival seems to be related to the level on the graft at which the transection occurs. ${ }^{1,2}$

An attempt was made to find a mechanism to take these transected hairs into consideration, but not to give them the full value of a non-transected hair. To do this, a "decreased survival" factor was arbitrarily assigned of $0.5(50 \%)$ to the transected hairs to create an "adjusted" hairs/graft parameter. After taking this into account, the adjusted hairs/graft decreased to 1.9 for the sharp punch and 2.2 for the blunt punch (Figure 4).

\begin{tabular}{|l|c|c|}
\hline \multicolumn{2}{|c|}{ Hairs per Graft } & \\
& Sharp & Blunt \\
\hline Hairs / graft & 2.6 & 2.5 \\
\hline Hairs / graft *(Adjusted for transection using a factor of 0.5) & 1.9 & 2.2 \\
\hline
\end{tabular}

Figure 4. Adjusted hairs per graft

\section{Yield/Missing Grafts}

Yield and missing grafts are two ways of looking at the same thing. Yield is the percent of extraction attempts that produce a graft. Missing grafts are the percent of extraction attempts that do not produce a graft. Missing grafts are most commonly felt to be due to a combination of capped and buried grafts, although there may be other unknown explanations.

Within the field of FUE, the issue of yield and missing grafts is most commonly discussed when referring to robotic graft extraction, because all attempts are counted during every procedure. In addition, physicians are charged for harvest attempts and not for the number of grafts they obtain, so it is an important financial issue for them. It has been less common to discuss yield and missing grafts in manual or motorized FUE for the simple reason that extraction attempts are not typically recorded. Because the design of this study consisted of doing exactly 200 attempts for both the sharp and dull, it was possible to measure the yield and missing grafts. It was determined that sharp had an overall slightly better yield than blunt (Figure 5). Out of 200 attempts, the sharp punch had a $93 \%$ yield (7\% missing grafts), while the blunt punch had an $87 \%$ yield (13\% missing grafts).

Surprisingly, the number of missing grafts due to capping missing grafts at $8 \%$ vs. $2 \%$ for the sharp. These missing grafts were assumed to have been buried grafts.

\begin{tabular}{|c|c|c|}
\hline \multicolumn{3}{|c|}{ Yield } \\
\hline & Sharp & Blunt \\
\hline Yield (out of 200 attempts) & $93 \%$ & $87 \%$ \\
\hline Capped grafts & $5 \%$ & $5 \%$ \\
\hline Yield (after taking into account capping) & $\underline{98 \%}$ & $\underline{92 \%}$ \\
\hline Missing grafts (buried, unknown) & $2 \%$ & $8 \%$ \\
\hline
\end{tabular}

Figure 5. Sharp vs. blunt punch yield rates

\section{Speed}

Many practitioners believe that the sharp punch has a slight speed of extraction advantage over the blunt punch. The data actually revealed that both techniques were similar in speed (Figure 6). The total time was divided into two steps: punching and pulling. With the sharp punch, the punching step was faster, while the pulling phase was slower. With the blunt punch, the opposite was noted with the punching phase being slower, while the pulling phase was faster. Overall, the total time was the same, and there was no benefit of one technique over the other with respect to speed.

\begin{tabular}{|l|c|c|}
\hline \multicolumn{2}{|c|}{ Speed of Extraction } \\
& Sharp & Blunt \\
\hline Punch time (minutes) & 9.4 & 11.1 \\
\hline Pull time (minutes) & 12.9 & 11.2 \\
\hline Extraction - total time (minutes) & 22 (537 grafts/hr) & 22 (540 grafts/hr) \\
\hline \multicolumn{2}{|c|}{ Figure 6. Sharp vs. blunt punch speed of extraction rates } \\
\hline
\end{tabular}

\section{Graft Quality}

Grafts from all patients were photographed and compared. In the majority of cases, slight differences were observed (Figure 7 ). The blunt grafts had slightly more tissue surrounding the follicles, and there appeared to be less of a separation or splaying of the follicles in the lower portions of the grafts. This may be significant as FUT studies have shown an improvement in the overall survival of grafts containing relatively more surrounding tissues. ${ }^{4,5}$ These benefits may be due to reducing the chances of dehydration and trauma, although the actual clinical significance of these differences in FUE grafts is unknown. 
Sharp vs. Blunt Punch from page 257

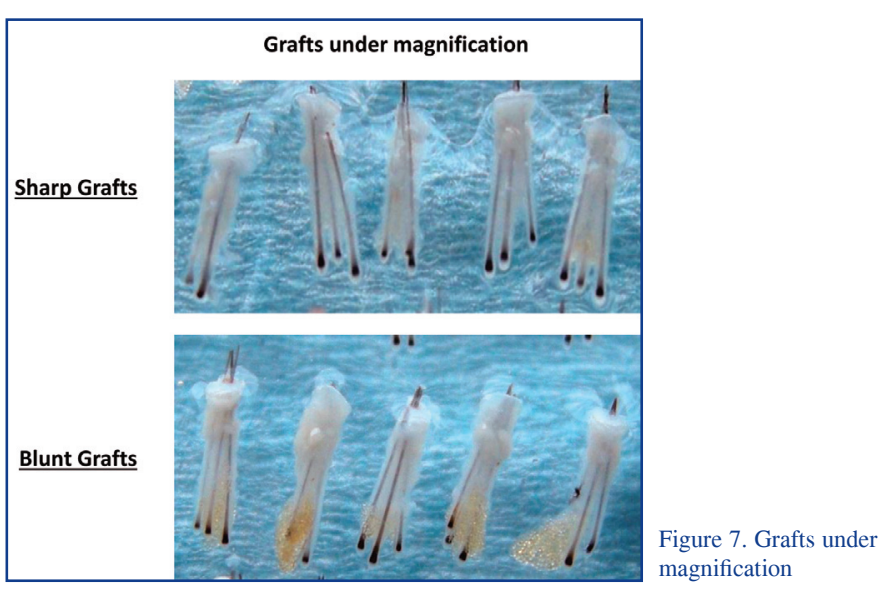

with sharp punch dissection, the lower the transection rates will be. The same holds true for a practitioner experienced with blunt punches. The only way to truly minimize this variable would be to have the world's best sharp punch practitioner extract one side and the world's best blunt punch practitioner extract the other side. Differences in transection between the two techniques would be less pronounced because the transection rates on both sides would be atypically better than occurs in the average clinical practice. More useful and clinically relevant data though is obtained from a study that compares the results when the same physician who is skilled in both techniques performs the extractions. Now, it is possible that for certain cases different sized punches and different instruments could have been used to give better results. The intent of the study was to use two punches of the same diameter to form a basis for comparison. That is a better reflection of what the potential differences are in the real world.

\section{Scarring}

The gross photographic views showed little difference between the sharp and blunt sides (Figure 8). No scarring was detectable on either side at a \#1 clipper guard haircut. When no guard was used, the small white dots typical of FUE extraction were visible but maintained an even and similar looking distribution on both sides.

At higher magnification, a subtle difference between the two sides was seen in some of the patients. The blunt side seemed to have a slightly patchier appearance compared to the sharp punch (Figure 9). A potential reason for this difference may be that the grafts on the blunt side had less transection and were more completely extracted. On the other hand, the grafts on the sharp side may have left behind some

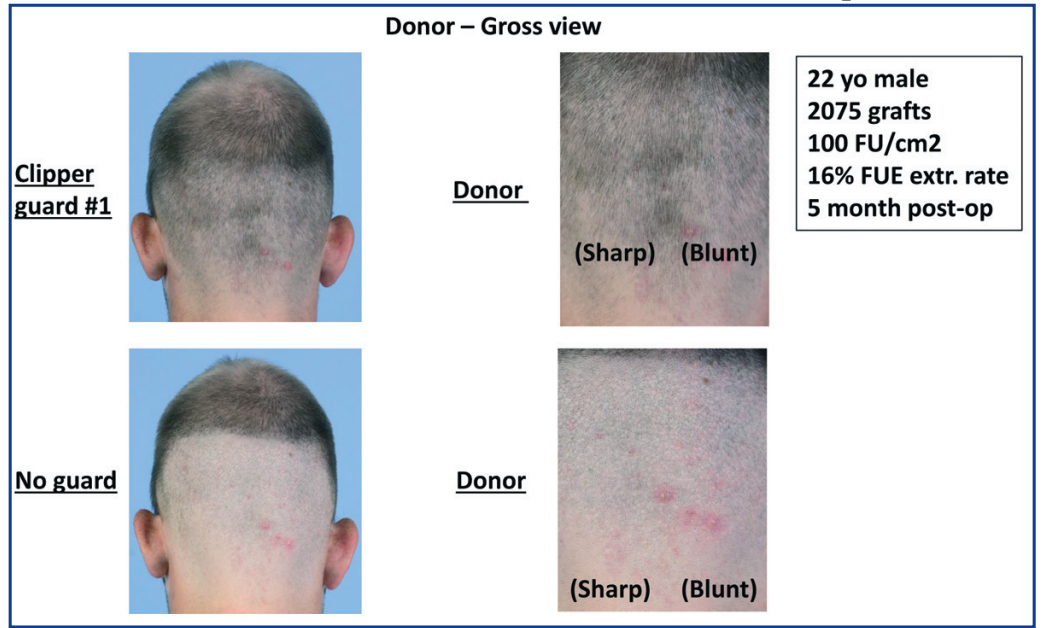

Figure 8. Graphic views showing little difference between the sharp and blunt sides.

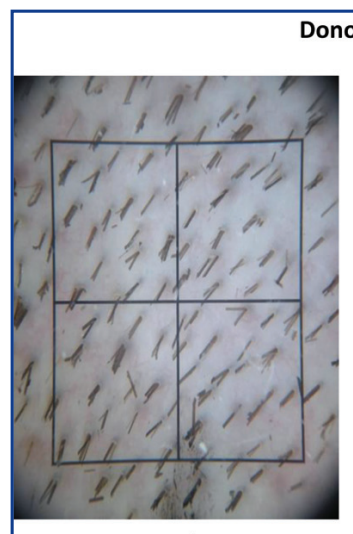

Sharp

Donor - Magnified view (3 month post-op)

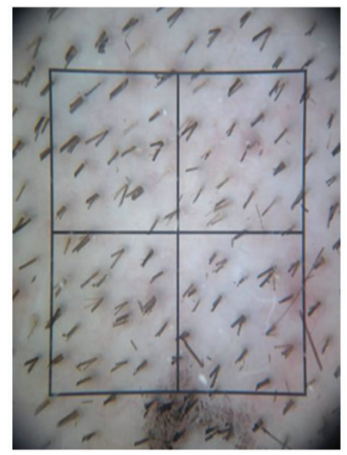

Blunt

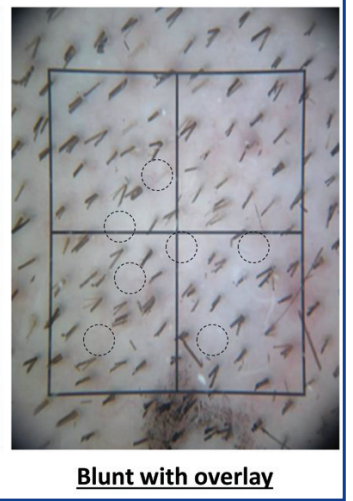

Blunt with overlay
Figure 9. Sharp and blunt sides at post-op 3 months; note patchier appearance on blunt side
Transection rates were higher with the sharp punch compared to the blunt punch $(\sim 20 \%$ sharp vs. $6 \%$ blunt). In addition, when looking at the type (level) of transection, more than $60 \%$ of the transactions occurred closer to the center of the hair. This location for transection is potentially significant at reducing regrowth of follicles according to a number of studies. $^{1-3}$

Another important observation was made during this part of the study that may have implications with respect to studies or transection rates reported from other clinics. It was discovered that in order to get accurate transection data, it was critical to have a "dedicated" assistant evaluating transection before the grafts were sorted or trimmed by other assistants. During the early phase of the study, extremely low transection rates were found. This was initially exciting, but also made the authors suspicious as the numbers were just too good to be true (i.e., $0 \%-1 \%$ for blunt and $4 \%-6 \%$ for sharp). The data was subsequently reanalyzed. It was discovered that in spite of explicit instructions on how to record transections, assistants who were multi-tasking (i.e., sorting, trimming, and counting transections) were making various errors. For example, sometimes they would trim away minor spicules that they felt were insignificant. Only after modifying the protocol and assigning a "dedicated" staff member to record all the transections she observed before any trimming and sorting was done, did more accurate and reliable results appear. It was then certain that every is indeed an important variable that could have influenced study's results. Obviously, the more experienced a physician is 


\section{Letters to the Editors}

Paul T. Rose, MD, JD, FISHRS Coral Gables, Florida USA

paultrose@yahoo.com

Re: Inventions and contributions to our specialty

I received the most recent edition of the Hair Transplant Forum and was delighted to see the article by Dr. Otavio Boaventura discussing how he performs long hair FUE (Long Hair FUE and the Donor Area Preview. 2016; 26(5):200-202). I also had the pleasure of meeting Dr. Boaventura during one of the ISHRS mini workshops where he discussed his impressive technique.

As many now know, he uses a slotted punch to perform the technique. After his lecture in a mini course, I related to him that I had demonstrated the use of a slot punch at the ISHRS meeting in 2007 and introduced the punch at a meeting in Greece. At that time, John Cole and I introduced the first limited depth control FUE/FIT punch (see photo). (Speaking

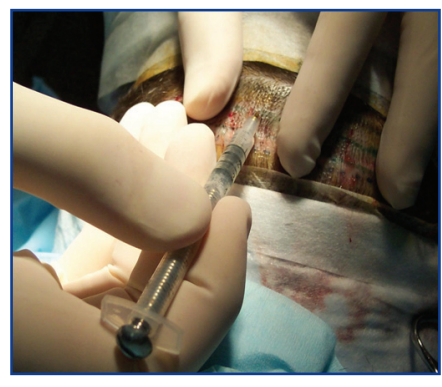

with Bill Rassman he indicated that he invented a limited depth device prior to John and I, but he never got around to publishing about it. Knowing Bill it certainly is possible.)

Subsequently, Dr. Boaventura included my name in his ISHRS presentation and gave me credit for invention of the slot punch although his use for long hair is quite different than my original intention to simply decrease transection. I wish to thank Dr. Boaventura for his integrity and kindness.

Additionally, I noted several instances in the meeting where people have, certainly unintentionally, taken credit for developing or inventing a technique or device that was created by someone else. I would urge our younger colleagues to try to carefully review the literature and perhaps inquire of some of the elder states-people in the field whether their discoveries have possibly been made by others previously. It is wonderful that we have a younger group of enthusiastic physicians entering our field who will no doubt make great contributions to the specialty. I think that we all realize that our accomplishments come from being educated by those who have preceded us, and they should be given credit for their efforts. transected hair was being counted. The rates were now slightly higher than some other clinics were reporting. How reliable are transection rates recorded by other clinics and in other studies where a "dedicated" staff member for data collection is not used?

The parameter of hairs/graft was very similar in both of these techniques with a slight edge toward the blunt punch (2.5 sharp vs. 2.6 blunt). There is a concern that if a clinic is only using the hairs/graft parameter to evaluate graft quality, there may be a false sense of security created because it ignores the transections within the grafts. For example, both sharp and blunt punches could produce a hair/graft ratio of approximately 2.4. According to this study though, with the sharp punch there was a greater number of transected hairs within those grafts. If you take into account these partial transected hairs as was also done in the study, the hairs/graft decreases (1.9 sharp and 2.2 blunt). This is not to say that hairs/graft is not important, but it should just not be the only parameter used when evaluating the overall success of FUE extraction. The significance of transection should not be ignored.

An obvious question remains: How important are these transections to the overall survival of the hairs in both the recipient and donor area? A number of studies have shown that transection can influence survival and growth to varying degrees in a negative way. ${ }^{1}$ Further studies in this area would be helpful in more completely answering that question.

Another observation was that the issue of decreased yield is not limited to just robotic FUE, but also occurs with both sharp and blunt motorized methods. In this study, the yield with the sharp punch was slightly better than the blunt punch $(93 \%$ sharp vs. $87 \%$ blunt). These were both superior than the average yield reported with earlier versions of the robot (as low as 75\%). At the time of this writing, newer versions of the robot have been released, and the yield may have improved. Capping is one of the causes of missing grafts and is highly correlated to the physician's skill in the under-appreciated step of manually removing (pulling) the FUE graft out of the scored incision. Buried grafts are another cause of missing grafts. When looking only at buried grafts, it appears that the blunt punch has an increased incidence of buried grafts compared to the sharp punch (8\% vs. $3 \%)$.

With respect to scarring, both sides looked similar trimmed with a \#1 guard clipper. However, with no guard and under higher magnification, the blunt punch side had a number of extraction sites with no hair, which gave a subtle patchy look. This was consistent with the theory that the sharp punch had left behind some hairs due to transection that may have regrown and contributed to a more even look. While, after only one session of FUE this may not cause any issues, subsequent surgeries may make it more difficult to cover the donor area with shorter hair. The questionable drawback of transected hair with the sharp punch may actually be of benefit to the patient in coverage of the donor area. Most patients who opt for FUE desire the ability to keep their hair short anyway and would prefer a less noticeable transplant.

\section{References}

1. Kim, J., and Y. Choi. Hair follicle regeneration after horizontal resectioning. In: D. Stough and R. Haber, eds. Hair Replacement: Surgical and Medical. St. Louis, MO: Mosby, 1996; 358-360.

2. Unger, W., M.L. Beehner, and M. Mayer. Studies comparing hair growth after transection through the bulge area to produce approximately two equal halves, graft survival, growth, and healing studies. In: W.P. Unger and R. Shapiro, eds. Hair Transplantation, 4th Ed. New York: Marcel Dekker, 2004.

3. Frechet, P., and M.L. Beehner. A multi-center study of the effect of $100 \%$ transection on hair graft survival. Presented at the 14th Annual Meeting of the ISHRS, San Diego, California, USA; 2006.

4. Beehner, M.L. A comparison of hair growth between follicular unit grafts trimmed "skinny" vs. "chubby." Hair Transplant Forum Int'l. 1999; 9:16.

5. Beehner, M.L. Comparison of survival of FU grafts trimmed chubby, medium, and skeletonized. Hair Transplant Forum Int'l. 2010; 20(1):1,6. 